\title{
The OntoWordNet Project: extension and axiomatization of conceptual relations in WordNet
}

\author{
Aldo Gangemi ${ }^{1}$, Roberto Navigli ${ }^{2}$, Paola Velardi ${ }^{2}$ \\ ${ }^{1}$ Laboratory for Applied Ontology, ISTC-CNR, \\ viale Marx 15, 00137 Roma, Italy \\ gangemi@ip.rm.cnr.it \\ 2 Dipartimento di Informatica, University of Roma "La Sapienza" \\ via Salaria 113, 00198 Roma, Italy \\ \{navigli,velardi\}@dsi.uniroma1.it
}

\begin{abstract}
In this paper we firstly present a progress report of the OntoWordNet project, a research program aimed at achieving a formal specification of WordNet. Within this program, we developed a hybrid bottom-up top-down methodology to automatically extract association relations from WordNet, and interpret these associations in terms of a set of conceptual relations, formally defined in the DOLCE foundational ontology. Preliminary results provide us with the conviction that a research program aiming to obtain a consistent, modularized, and axiomatized ontology from WordNet can be completed in acceptable time with the support of semi-automatic techniques.
\end{abstract}

\section{Introduction}

The number of applications where WordNet (WN) is being used as an ontology rather than as a mere lexical resource seems to be ever growing. Indeed, WordNet contains a good coverage of both the lexical and conceptual palettes of the English language. However, WordNet is serviceable as an ontology (in the sense of a theory expressed in some logical language) if some of its lexical links are interpreted according to a formal semantics that tells us something about the way we use a lexical item in some context for some purpose. In other words, we need a formal specification of the conceptualizations that are expressed by means of WordNet's synsets ${ }^{1}$. A formal specification requires a clear semantics for the primitives used to export WordNet information into an ontology, and a methodology that explains how WordNet information can be bootstrapped, mapped, refined, and modularized during the export procedure.

The formal specification of WordNet is the objective of the so-called OntoWordNet research program, started two years ago at the ISTC-CNR, and now being extended with other partners, since collaborations have been established with the universities of Princeton, Berlin and Roma. The program is detailed in section 2, where we outline the main objectives and current achievements.

In this paper we describe a joint ongoing work of ISTC-CNR and the University of Roma that has produced a methodology and some preliminary results for adding axioms (DAML+OIL "restrictions") to the concepts derived from WordNet synsets. The methodology is hybrid because it employs both top-down techniques and tools from formal ontology, and bottom-up techniques from computational linguistics and machine learning. Section 3 presents a detailed description of the methodology.

The preliminary results, presented in section 4, seem very encouraging, and provide us with the convincement that a research program aiming to obtain a consistent, modularized, and axiomatized ontology from WordNet can be completed in acceptable time with the support of semi-automatic techniques.

\footnotetext{
${ }^{1}$ Concept names in WordNet are called synsets, since the naming policy for a concept is a set of synonym words, e.g. for sense 1 of car: $\{$ car, auto, automobile, machine, motorcar $\}$. In what follows, WN concepts are also referred to as synsets.
} 


\section{The OntoWordNet research program: objectives, assumptions, and first achievements}

The OntoWordNet project aims at producing a formal specification of WordNet as an axiomatic theory (an ontology). To this end, WordNet is reorganized and enriched in order to adhere to the following commitments:

- Logical commitment. WordNet synsets are transformed into logical types, with a formal semantics for lexical relations. The WordNet lexicon is also separated from the logical namespace.

- Ontological commitment. WordNet is transformed into a general-purpose ontology library, with explicit categorial criteria, based on formal ontological distinctions (Gangemi et al. 2001). For example, the distinctions enable a clear separation between concept-synsets, instance-synsets, relation-synsets, and metaproperty-synsets. Moreover, such formal ontological principles facilitate the axiomatic enrichment of the ontology library.

- Contextual commitment. WordNet is modularized according to knowledge-oriented domains of interest. The modules constitute a partial order.

- Semiotic commitment. WordNet lexicon is linked to text-oriented (or speech act-oriented) domains of interest, with lexical items ordered by preference, frequency, combinatorial relevance, etc.

The logical commitment has been already introduced in WordNet through methodological assumptions that are described in (Gangemi et al. 2002). The hyperonymy relation in WN is basically interpreted as formal subsumption, although hyperonymy for concepts referring to individuals (geographical names, characters, some techniques, etc.) is interpreted as instantiation. This will be referred as assumption A1 ("hyperonymy as synset subsumption"). For example, the concept retrospective\#1 has the hyperonym art_exhibition\#1, which is logically represented as:

$$
\forall x \text {. Retrospective }(x) \rightarrow \text { Art_Exhibition }(x),
$$

while the hyperonymy link between the gemini\#1 and constellation\#1 is represented as an instantiation:

\section{Constellation(Gemini)}

The ontological commitment is rather more demanding, but many results are already available. For example, an incremental methodology has been adopted, reusing the DOLCE foundational ontology (Gangemi et al. 2002), in order to revise or to reorganize WordNet synset taxonomies and relations (see also paragraph 3.2.1). Substantial work has been done on the refinement of the hyponym/hyperonym relation, which has being investigated since several years. WordNet synonymy is a relation between words, not concepts, therefore we should assume that the synonymy relation (synsets in WordNet) is an equivalence class of words (or phrases), sharing the same meaning within an ontology. Consequently, two words are synonyms when their intended meaning in WordNet is the same. This will be referred to as assumption A2 ("synset as meaning equivalence class").

However, we have no formal definition of words in WordNet that allows us to create equivalence classes (synsets) analytically (i.e., to state semantic equivalences). Instead, we have pre-formal synsets that have been validated by lexicographers with an intuition that could be formalized as semantic equivalence. Part of this intuition is conveyed by textual definitions (called glosses). No claim of completeness is made though. This will be referred as assumption A3 ("glosses as axiomatizations"). In this paper we are trying to formalize such intuition.

A related assumption that we make is that words in glosses are used in a way consistent to the WordNet synsets. This will be referred as assumption A4 ("glosses are synset-consistent"). A4 lets us assume also that the informal theory underlying synsets, hyperonymy relations, and glosses, can be formalized against a finite signature (the set of WN synsets), and a set of axioms derived from the associations (A-links) between any synset $S$ and the synsets that can be associated to the words used in the gloss of $S$. This is dependent on A3 and A4, and may be referred as assumption A5 ("A-links as conceptual relations").

The revision of WordNet synset taxonomies is still ongoing, but it is already usable to carry out novel experiments. For example, the WEBKB- $2^{2}$ project is using the preliminary results of our work.

Contextual and semiotic commitments are still poorly implemented, although some resources and the methodologies to exploit them are available. For example, contextual information could be extracted using the so-called domain labels defined in (Miller et al. 1993) and (Magnini and Cavaglia, 2000). Domain labels have been associated to WordNet 1.6 synset, and we are currently analyzing and refining this information.

\footnotetext{
${ }^{2}$ http://meganesia.int.gu.edu.au/ phmartin/WebKB/doc/wn
} 
For example, domain labels are being exploited in order to create a partial order of ontological modules that is consistent with the actual use of the lexicon within real world corpora. To this purpose, we are using both foundational ontologies (top-down reorganization), and Web catalogues (bottom-up reorganization).

Figure 1 shows the "layers" in which the OntoWordNet ontology library is being organized. The foundational layer contains modules including domain-independent concepts, relations, and meta-properties. The core layer contains modules including generic concept and relations for a given domain of interest. The domain layer contains modules including domain-oriented instances, concepts, and relations. This layer can be automatically populated by an ontology extension technique, implemented in the OntoLearn system (Navigli et al. 2003).

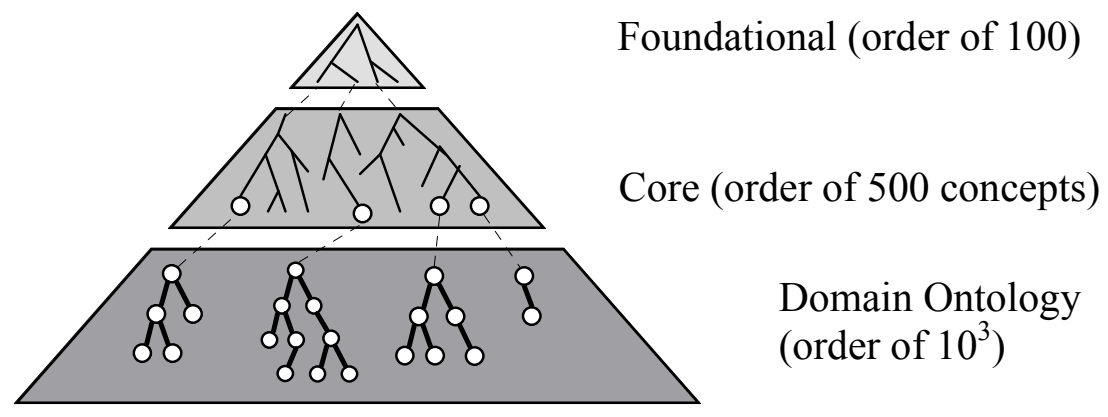

Figure 1. The three levels of generality of a Domain Ontology.

\section{Axiomatization of WordNet: Extraction and refinement of association sets from glosses}

The task of axiomatizing WordNet, starting from assumptions A1-A5 outlined in the previous section, requires that the informal definition in a synset gloss be transformed in a logical form. To this end, first, words in a gloss must be disambiguated, i.e. replaced by their appropriate synsets. This first step provides us with pairs of generic semantic associations (A-links) between a synset and the synsets of its gloss. Secondly, A-links must be interpreted in terms of more precise, formally defined semantic relations. The inventory of semantic relations is selected or specialized from the foundational ontology DOLCE, as detailed later, since in WordNet only a limited set of relations are used, that are partly ontological, partly lexical in nature. For example, part_of (meronymy in WordNet) and kind_of (hyponymy in WordNet) are typical semantic relations, while antonymy (e.g. liberal and conservative) and pertonymy (e.g slow and slowly) are lexical relations. Furthermore, WordNet relations are not axiomatized, nor are they used in a fully consistent way.

To summarize, the objective of the method described in this section is to:

- $\quad$ automatically extract a number of semantic relations implicitly encoded in WordNet, i.e. the relations holding between a synset and the synsets in its gloss.

- (semi)-automatically interpret and axiomatize these relations.

For example, sense 1 of driver has the following gloss "the operator of a motor vehicle". The appropriate sense of operator is \#2: operator, manipulator ("an agent that operates some apparatus or machine"), while motor vehicle is monosemous: motor vehicle, automotive vehicle ("a self-propelled wheeled vehicle that does not run on rails").

After automatic sense disambiguation, we (hopefully) learn that there exists an A-link between driver\#1 and operator\#2, and between driver\#1 and motor vehicle\#1. Subsequently, given a set of axiomatized semantic relations in DOLCE, we must select the relation that best fits the semantic restrictions on the relation universes (domain and co-domain, or range). For example, given an A-link between driver\#1 and motor vehicle\#1, the best fitting relation is agentive-coparticipation (Figure 2), whose definition is:

(and co-participation

(domain Agentive_Physical_Object)

(range Non_Agentive_Functional_Object)) $)^{3}$.

\footnotetext{
${ }^{3}$ In FOL: AG_CO_PCP $(x, y)=$ df CO_PCP $(x, y) \wedge$ Agentive_Physical_Object $(x) \wedge$ Non_Agentive_Functional_Object $(y)$
} 


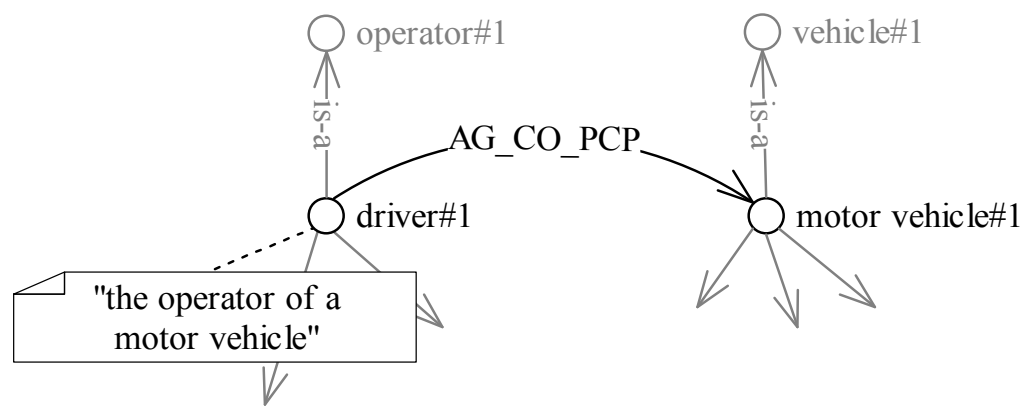

Figure 2. An example of semantic relation.

Domain and range in a conceptual relation definition are established in terms of the DOLCE ontology. Consequently, another necessary step of our method is to re-link at least some of the higher level nodes in WordNet with the DOLCE upper ontology.

In the following sub-sections we detail the procedures for gloss disambiguation, WordNet re-linking, and selection of conceptual relations.

\subsection{Bottom-up learning of association links.}

The first step is a bottom-up procedure that analyses the NL definitions (glosses) in WordNet and creates the A-links. For each gloss (i.e., linguistic concept definition), we perform the following automatic tasks:

a) POS-tagging of glosses (using the ARIOSTO NL processor) and extraction of relevant words;

b) Disambiguation of glosses by the algorithm described hereafter;

c) Creation of explicit "association" links (A-links) from synsets found in glosses to synsets to which glosses belong.

\subsubsection{Description of the gloss disambiguation algorithm}

We developed a greedy algorithm for gloss disambiguation that relies on a set of heuristic rules and is based on multiple, incremental iterations. A simplified formal description of the algorithm is in Figure 3.

The algorithm takes as input the synset $S$ whose gloss $G$ we want to disambiguate.

Two sets are used, $P$ and $D . D$ is a set of disambiguated synsets, initially including only the synset S. $P$ is a set of terms to be disambiguated, initially containing all the terms from gloss $G$ and from the glosses $\left\{G^{\prime}\right\}$ of the direct hyperonyms of $S$. As clarified later, adding $\left\{G^{\prime}\right\}$ provides a richer context for semantic disambiguation. The term list is obtained using our NL processor to lemmatize words, and then removing irrelevant words. We use standard information retrieval techniques (e.g stop words) to identify irrelevant terms.

When, at each iteration of the algorithm, we disambiguate some of the terms in $P$, we remove them from $P$ and add their interpretation (i.e. synsets) to the set $D$. Thus, at each step, we can distinguish between pending and disambiguated terms (respectively the sets $P$ and $D$ ). Notice again that $P$ is a set of terms, while $D$ contains synsets.

\section{a) Find monosemous terms}

The first step of the algorithm is to remove monosemous terms from $P$ (those with a unique synset) and include their unique interpretation in the set $D$.

\section{b) Disambiguate polysemous terms}

Then, the core iterative section of the algorithm starts. The objective is to detect semantic relations between some of the synsets in $D$ and some of the synsets associated to the terms in $P$. Let $S^{\prime}$ be a synset in $D$ (an already chosen interpretation of term $t^{\prime}$ ) and $S^{\prime \prime}$ one of the synsets of a polysemous term $t^{\prime \prime} \in P$ (i.e., $t$ " is still ambiguous). If a semantic relation is found between $S^{\prime}$ and $S$ ", then $S^{\prime \prime}$ is added to $D$ and $t^{\prime \prime}$ is removed from $P$.

To detect semantic relations between $S^{\prime}$ and $S^{\prime \prime}$, we apply a set of heuristics grouped in two classes, Path and Context, described in what follows. Some of these heuristics have been suggested in (Milhalcea, 2001),

\section{Path heuritics}

The heuristics in class Path seek for semantic patterns between the node $S^{\prime}$ and the node $S^{\prime \prime}$ in the WordNet semantic network. A pattern is a chain of nodes (synsets) and arcs (directed semantic relations), where $S^{\prime}$ and $S^{\prime \prime}$ are at the extremes. 
Formally, we define $S^{\prime} \stackrel{R}{\rightarrow}^{n} S^{\prime \prime}$ as $S^{\prime} \stackrel{R}{\rightarrow}^{S_{1}} \stackrel{R}{\rightarrow} \ldots \stackrel{R}{\rightarrow} S_{n} \equiv S^{\prime \prime}$, that is a chain of $n$ instances of the relation $R$. We also define $\stackrel{R_{1}, R_{2}}{\rightarrow}$ as $\stackrel{R_{1}}{\rightarrow} \cup \stackrel{R_{2}}{\rightarrow}$.

(a) $~ \# \quad \% \quad \&$

The symbols: $\rightarrow, \rightarrow, \rightarrow, \rightarrow, \rightarrow$ respectively represent the following semantic relations coded in WordNet 1.6: hyperonimy (kind_of), hyponymy (has kind) meronymy (part_of), holonymy (has_part), and similarity. Similarity is a generic relation including near synonyms, adjectival clusters and antonyms. Finally, the gloss relation $S \stackrel{\text { gloss }}{\rightarrow} T$ indicates that the gloss of $S$ includes a term $t$, and $T$ is one of the synsets of $t$.

We use the following heuristics to identify semantic paths $\left(S^{\prime} \in D, S^{\prime \prime} \in \operatorname{Synsets}\left(t^{\prime \prime}\right), t^{\prime \prime} \in P\right)$ :

1 Hyperonymy path: if $S^{\prime} \rightarrow^{n} S^{\prime \prime}$ choose $S^{\prime \prime}$ as the right sense of $t^{\prime \prime}$ (e.g., canoe\#1 $\stackrel{@}{ }^{2}$ boat\#l, i.e. a canoe is a kind of boat);

2 Hyperonymy/Meronymy path: if $S^{, ~} \rightarrow^{\circledR},{ }^{\#} S^{\prime \prime}$ choose $S$ ” as the right sense of $t^{\prime \prime}$ (e.g., archipelago\#1 $\stackrel{\#}{\rightarrow}$ island\#1);

3 Hyponymy/Holonymy path: if $S^{\prime} \rightarrow^{\rightarrow} S^{\prime \prime}$ choose $S^{\prime \prime}$ as the right sense of $t$ " (e.g., window\#7 $\stackrel{\%}{\rightarrow}$ computer screen\#1);

4 Adjectival Similarity: if $S^{\prime \prime}$ is in the same adjectival cluster than $S^{\prime}$, choose $S^{\prime \prime}$ as the right sense of $t$ ”.

5 Parallelism: if exists a synset $T$ such that $S \stackrel{@}{\rightarrow} T \stackrel{@}{\leftarrow} S$ ”, choose $S^{\prime \prime}$ as the right sense of $t$ ” (for example, background\#1 $\stackrel{@}{\rightarrow}$ scene\#3 $\stackrel{@}{\leftarrow}$ foreground\#2);

\section{Context heuristics}

The Context heuristics use several available resources to detect co-occurrence patterns in sentences and contextual clues to determine a semantic proximity between $S$ ' and $S$ '. The following heuristics are defined:

1 Semantic co-occurrences: word pairs may help in the disambiguation task if they always co-occur with the same senses within a tagged corpus. We use three resources in order to look for co-occurrences, namely:

- the SemCor corpus, a corpus where each word in a sentence is assigned a sense selected from the WordNet sense inventory for that word; an excerpt of a SemCor document follows:

Color\#1 was delayed\#1 until 1935, the widescreen\#1 until the early\#1 fifties\#1. Movement\#7 itself was\#7 the chief\#1 and often\#1 the only\# attraction\#4 of the primitive\#1 movies\#1 of the nineties\#1.

- the $L D C$ corpus, a corpus where each document is a collection of sentences having a certain word in common. The corpus provides a sense tag for each occurrence of the word within the document. Unfortunately, the number of documents (and therefore the number of different tagged words) is limited to about 200. An example taken from the document focused on the noun house follows:

Ten years ago, he had come to the house\#2 to be interviewed.

Halfway across the house\#1, he could have smelled her morning perfume.

- gloss examples: in WordNet, besides glosses, examples are sometimes provided containing synsets rather than words. From these examples, as for the LDC Corpus, a co-occurrence information can be extracted. With respect to the LDC corpus, WordNet provides examples for thousands of synsets, but just a few for the same word. Some examples follow:

"Overnight accommodations\#4 are available."

"Is there intelligent\#1 life in the universe?"

"An intelligent\#1 question."

As we said above, only the SemCor corpus provides a sense for each word in a pair of adjacent words occurring in the corpus, while LDC and gloss examples provide the right sense only for one of the terms.

In either case, we can use this information to choose the synset $S$ " as the interpretation of $t$ " if the pair $t$ ' $t$ " occurs in the gloss and there is an agreement among (at least two of) the three resources about the disambiguation of the pair $t^{\prime} t$ '. For example:

[...] Multnomah County may be short of general assistance money in its budget to handle an unusually high summer\#1 month\#1's need [...]. 
Later\#1, Eckenfelder increased\#2 the efficiency\#1 of treatment\#1 to between 75 and 85 percent\#1 in the summer\#1 months\#1.

are sentences respectively from the LDC Corpus and SemCor. Since there is a full agreement between the resources, one can easily disambiguate summer and months in the gloss of summer_camp\#1: " $a$ site where care and activities are provided for children during the summer months".

2 Common domain labels: Domain labels are the result of a semiautomatic methodology described in (Magnini and Cavaglia, 2000) for assigning domain labels (e.g. tourism, zoology, sport..) to WordNet synsets ${ }^{4}$. This information can be exploited to disambiguate those terms with the same domain labels of the start synset $S$. Notice that a synset can be marked with many domain labels, therefore the algorithm selects the interpretation $S$ " of $t$ " if the following conditions hold together (the factotum label is excluded because it is a sort of topmost domain):

- DomainLabels $\left(S^{\prime \prime}\right) \backslash\{$ factotum $\} \subseteq$ DomainLabels $(S) \backslash\{$ factotum $\}$;

- There is no other interpretation $S^{\prime \prime}$ of $t$ " such that DomainLabels $\left(S^{\prime \prime}\right) \backslash\{$ factotum $\} \subseteq$ DomainLabels $(S) \backslash\{$ factotum $\}$.

For example, boat\#1 is defined as "a small vessel for travel on water", both boat\#1 and travel\#1 belong to the tourism domain and no other sense of travel satisfies the conditions, so the first sense of travel can be chosen; similarly, cable car\#1 is defined as "a conveyance for passengers or freight on a cable railway", both cable car\#1 and conveyance\#1 belong to the transport domain and no other sense of conveyance satisfies the conditions, so the first sense of conveyance is selected.

\section{c) Update $D$ and $P$}

During each iteration, the algorithm applies all the available heuristics in the attempt of disambiguating some of the terms in $P$, using all the available synsets in $D$. While this is not explicit in the simplified specification of Figure 3 , the heuristics are applied in a fixed order reflecting their importance, that has been experimentally determined. For example, Context heuristics are applied after Path heuristics 1-5. At the end of each iterative step, new synsets are added to $D$, and the correspondent terms are deleted from $P$. The next iteration makes use of these new synsets in order to possibly disambiguate other terms in $P$. Eventually, either $P$ becomes empty, or no new semantic relations can be found.

When the algorithm terminates, $D \backslash\{S\}$ can be considered a first approximation of a semantic definition of $S$. For mere gloss disambiguation purposes, the tagged terms in the hyperonyms' gloss are discarded, so that the resulting set (GlossSynsets) now contains only interpretations of terms extracted from the gloss of $S$. At this stage, we can only say that there is a semantic relation (A-link) between $S$ and each of the synsets in GlossSynsets .

A second, more precise approximation of a sound ontological definition for $S$ is obtained by determining the nature of the A-links connecting $S$ with each concept in $D \backslash\{S\}$. This is an ongoing task and is discussed in Section 4 .

\subsubsection{A running example}

In the following, we present a sample execution of the algorithm on sense 1 of retrospective. Its gloss defines the concept as "an exhibition of a representative selection of an artist's life work", while its hyperonym, art exhibition\#1, is defined as "an exhibition of art objects (paintings or statues)". Initially we have:

$D=\{$ retrospective\#1 $\}$

$P=\{$ work, object, exhibition, life, statue, artist, selection, representative, painting, art $\}$

The application of the monosemy step gives the following result:

$D=\{$ retrospective\#1, statue\#1, artist $\# 1\}$

$P=\{$ work, object, exhibition, life, selection, representative, painting, art $\}$

because statue and artist are monosemous terms in WordNet. During the first iteration, the algorithm finds three matching paths:

retrospective\#1 $\stackrel{9}{2}^{2}$ exhibition\#2, statue\#1 $\stackrel{@}{\rightarrow}^{3}$ art\#1 and statue\#1 $\stackrel{9}{\rightarrow}^{6}$ object\#1

this leads to:

\footnotetext{
${ }^{4}$ Domain labels have been kindly made available by the IRST to our institution for research purposes.
} 
$D=\{$ retrospective\#1, statue $\# 1$, artist $\# 1$, exhibition $\# 2$, object $\# 1$, art $\# 1\}$

$P=\{$ work, life, selection, representative, painting $\}$

\section{DisambiguateGloss(S)}

$\{$ G already disambiguated? \}

if $($ GlossSynset $(S) \neq \varnothing)$ return

$\{\mathrm{S}$ is the starting point $\}$

$\mathrm{D}:=\{\mathrm{S}\}$

$\{$ disambiguation is applied the terms within the gloss of S and the glosses of its direct hyperonyms

$\mathrm{P}:=\operatorname{Gloss}(\mathrm{S}) \cup \operatorname{Gloss}(\operatorname{Hyper}(\mathrm{S}))$

\{look for synsets associated to monosemous terms in $\mathrm{P}$ \}

$\mathrm{M}:=$ SynsetsFromMonosemousTerms $(\mathrm{P})$

$\mathrm{D}:=\mathrm{D} \cup \mathrm{M}$

\{ 'Terms' returns the terms contained in the gloss of $\mathrm{M}$ \}

$\mathrm{P}:=\mathrm{P} \backslash$ Terms(M)

LastIteration: $=\mathrm{D}$

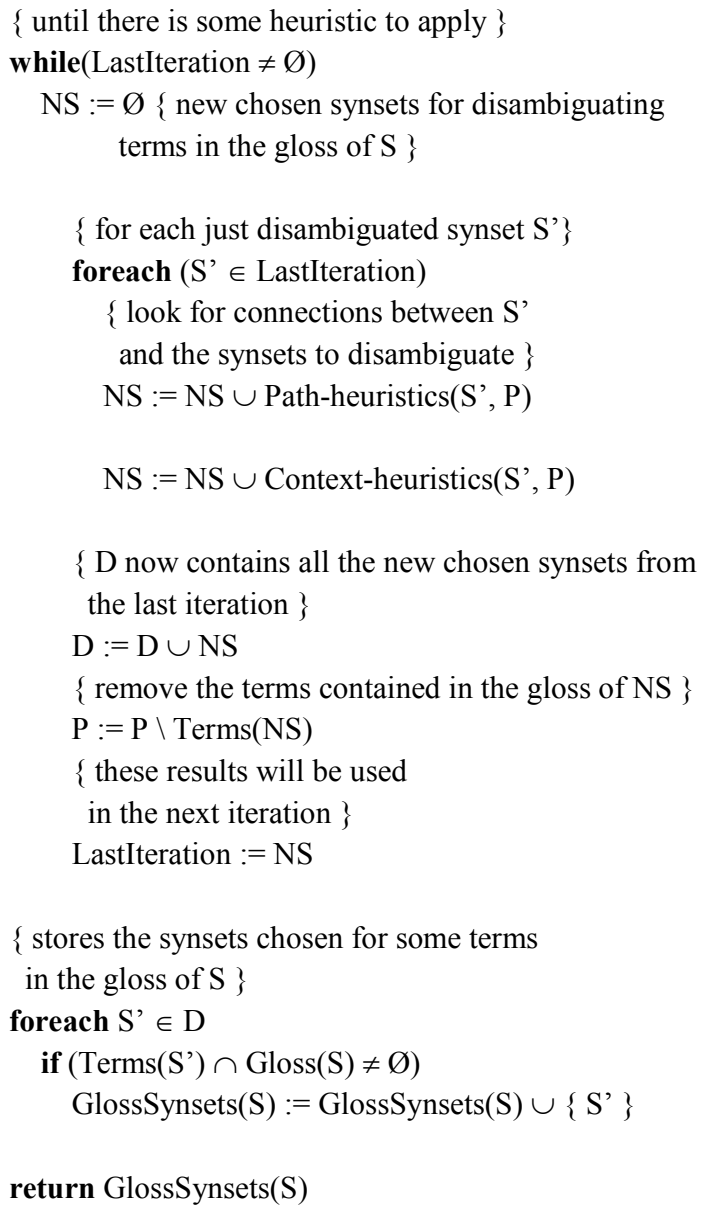

\section{Figure 3. The disambiguation algorithm.}

During the second iteration, an hyponymy/holonymy path is found:

$\operatorname{art} \# 1 \sim^{2}$ painting\#1 (painting is a kind of art)

$D=\{$ retrospective\#1, statue\#1, artist $\# 1$, exhibition\#2, object\#1, art $\# 1$, painting \#1 $\}$

$P=\{$ work, life, selection, representative $\}$

Since no new paths are found, the third iteration makes use of the LDC Corpus to find the co-occurrence "artist life", with sense 12 of life (biography, life history):

$D=\{$ retrospective\#1, statue\#1, artist\#1, exhibition\#2, object\#1, art\#1, painting\#1, life\#12 \}

$P=\{$ work, selection, representative $\}$

Notice that, during an iteration, the context heuristics are used only if the path heuristics fail.

The algorithm stops because there are no additional matches. The chosen senses concerning terms contained in the hyperonym's gloss were of help during disambiguation, but are now discarded. Thus we have:

GlossSynsets(retrospective\#1) $=\{$ artist $\# 1$, exhibition\#2, life\#12 $\}$ 


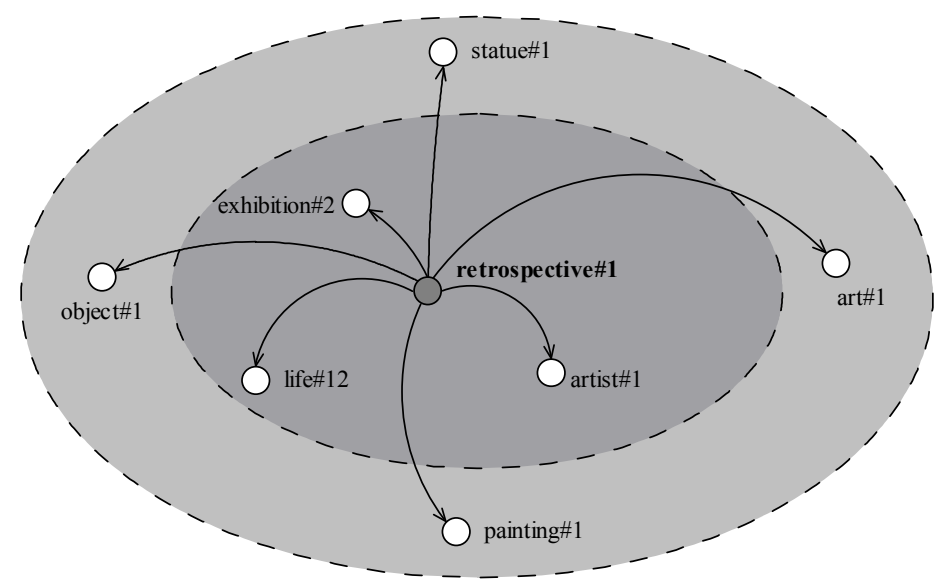

Figure 4. A first approximation of a semantic definition of retrospective\#1.

Figure 4 shows in dark gray the A-links between retrospective\#1 and the synset of its glosses, while in the light gray area are shown the synsets of the hyperonyms.

\subsection{Top-down learning: formal ontologies and WordNet "sweetening"}

In the top-down phase, the A-links extracted in the bottom-up phase are refined. A-links are similar to RT (Related Term) relations in thesauri, which provide just a clue of relatedness between pairs of thesaurus descriptors ${ }^{5}$. In fact, associations are conceptually ambiguous, since we can only assume that there is some relatedness between a synset and another synset extracted from the gloss analysis, but this relatedness must be explicit, in order to understand if it is a hyperonymy relation, or some other conceptual relation (e.g. part, participation, location, etc.).

First of all, we need a shared set of conceptual relations to be considered as candidates for A-links explicitation, otherwise the result is not easily reusable. Secondly, these relations must be formally defined. In fact, as already pointed out at the beginning of section 3, not only are A-links vague, but they also lack of a formal semantics: for example, if we decide (which seems reasonable) to represent associations as binary relations -like DAML+OIL "properties"- is an association symmetric? Does it hold for every instance, or only for some of the instances of the classes derived from the associated synsets? Is it just a constraint on the applicability of a relation to that pair of classes? Is the relation set a flat list, or there is a taxonomic ordering?

To answer such questions, the shared set of relations should be defined in a logical language using a formal semantics. Since WordNet is a general-purpose resource, the formal shared set of relations should also be general enough, based on domain-independent principles, but still flexible, in order to be easily maintained and negotiated.

\subsubsection{The DOLCE descriptive ontology and conceptual relation library}

A proposal in this direction is provided by the WonderWeb ${ }^{6}$ project Foundational Ontology Library (WFOL), which will contain a library including both compatible and alternative modules with domain-independent concepts and relations. A recently defined module that accomplishes the abovementioned requirements is DOLCE (Descriptive Ontology for Linguistic and Cognitive Engineering).

DOLCE is expressed in an S5 modal logic (Masolo et al. 2002), and has counterparts in computational logics, such as KIF, LOOM, RACER, DAML+OIL, and OWL. The non-KIF counterparts implement a less rich version, called DOLCE-Lite. DOLCE-Lite has been extended with some generic plugins for representing information, communication, plans, ordinary places, and with some domain plugins for representing e.g. legal, tourism, biomedical notions. The combination of DOLCE-Lite and the existing plugins is called DOLCE-Lite+. The current version 3.6 of, DOLCELite+ without domain plugins contains more than 300 concepts and about 150 relations (see table 1 and 2 in Appendix $1)$.

DOLCE assumes that its categories (top classes) constitute an extensionally closed set on any possible particular entity, i.e., entities that cannot be further instantiated within the assumptions of the theory (cf. Masolo et al. 2002, Gangemi et al. 2001). Of course, DOLCE does not assume an intensionally closed set, thus allowing for alternative ontologies to coexist. Such assumptions will be referred to as $A 6_{-} D$ ("extensional total coverage of DOLCE"). Consequently, we also

\footnotetext{
${ }^{5}$ A-links have an advantage over RT relations, because A-links are directed, while RT are symmetric relations. A-links are directed because we assume that the links can hold only from a source synset to a synset extracted from its gloss.

${ }^{6}$ http://wonderweb.semanticweb.org
} 
assume that WN globally can be tentatively considered a (extensional) subset of DOLCE, after its formalization. Since we cannot practically obtain a complete formalization of $\mathrm{WN}$, we will be content with incrementally approximating it. A trivial formalization of WN might consist in declaring formal subsumptions for all unique beginners under DOLCE categories, but this proved to be impossible, since the intension of unique beginners, once they are formalized as classes, is not consistent with the intension of DOLCE categories. Then we started (Gangemi et al. 2002) deepening our analysis of WN synsets, in order to find synsets that could be subsumed by a DOLCE category (or one of their subclasses) without being inconsistent.

In our previous OntoWordNet work, WordNet 1.6 has been analyzed, and 809 synsets have been relinked to DOLCELite+ in order to harmonize ("sweeten") WN taxonomies with DOLCE-Lite+. A working hypothesis $\left(A 7 \_D\right)$ has been that the taxonomy branches of the relinked synsets are ontologically consistent with the DOLCE-Lite + concepts, to which the relinking is targeted. This hypothesis resulted inadequate in the initial attempts to get a complete DOLCE coverage of WordNet, since the intended meanings of hyponym synsets are usually not consistent through the entire branching (cf. Gangemi et al. 2002 for examples) After some additional work, the current linking of 809 synsets seems acceptable, but it still needs refinements, in order to keep DOLCE-Lite+ intended meanings consistent through the overall WordNet branchings.

This task is still ongoing: some subsumptions are debatable, and it must be considered that some extensions of DOLCELite + are still unstable. Nonetheless, such an approximate and partly debatable coverage could be enough to start experimenting with a more explicit axiomatization of synsets. We will show in what follows that this experiment can also provide feedback to refine some of the subsumptions.

\subsubsection{Disambiguation of association links}

Assumptions A4 and A5 (section 2), together with A6_D (in previous sub-section), make it possible to exploit the axiomatized relations in DOLCE-Lite+. Such relations are formally characterized by means of ground axioms (e.g. symmetry, transitivity, etc.), argument restrictions (qualification of their universe), existential axioms, links to other primitives, theorems, etc. (refer to (Masolo et al. 2002), and the web site of the $\mathrm{LOA}^{7}$ ).

By looking at the A-links, a human expert can easily decide which relation from DOLCE-Lite+ is applicable in order to disambiguate the A-link, for example, from:

$$
\text { 1. A-link(car\#1, engine\#1) }
$$

we may be able to infer that cars have engines as components:

$$
\forall x . \operatorname{Car}(x) \rightarrow \exists y \text {. Engine }(y) \wedge \operatorname{Component}(x, y)
$$

or that from

\section{A-link(art_exhibition\#1, painting\#1)}

we can infer that exhibitions as collections have paintings as members:

$$
\forall x \text {. Art_exhibition }(x) \rightarrow \exists y \text {. Painting }(y) \wedge \operatorname{Member}(x, y)
$$

But this is an intellectual technique that requires a lot of effort. We are instead interested, at least for the sake of bootstrapping a preliminary axiomatization of synsets, in a (semi) automatic classification technique.

From this viewpoint, the only available structure is represented by the concepts (synsets) to which the A-links apply. Such synsets can be assumed as the argument restrictions of a conceptual relation implicit in the association. For example, from (A- $\left.\operatorname{link}\left(S_{1}, S_{2}\right)\right)$, we can infer that $\mathrm{R}_{\text {ass }}(x, y) \rightarrow \mathrm{S}_{1}(x) \wedge \mathrm{S}_{2}(y)$. Then, from A5 and its depend-on assumptions, we have a good heuristics for concluding that $\mathrm{S}_{1}(x) \rightarrow \exists y . \mathrm{R}_{\text {ass }}(x, y) \wedge \mathrm{S}_{2}(y)$. But this leaves us with the question of what is the intension of $\mathrm{R}_{\text {ass }}(x, y)$, beyond its argument restrictions.

In fact, assuming A6_ $\mathrm{D}$, this task is equivalent to asserting that $\mathrm{R}_{\text {dolce }}(x, y)$ subsumes $\mathrm{R}_{\text {ass }}(x, y)$. This assertion is also easily derivable, since a description-logic classifier (e.g. LOOM, MacGregor, 1993, or RACER, Moeller, 2001) can be used to compute the applicable relations from DOLCE-Lite+ to the training set of A-links, by using an "ABox" query like the following:

\section{ABox-1}

(retrieve (?x ?R ?y) (and (get-role-types ?x ?R ?y) (min-cardinality ?x ?R 1) (A-link ?x ?y)))

\footnotetext{
${ }^{7}$ http://ontology.ip.rm.cnr.it
} 
i.e., provided that A-links have been defined on DOLCE-Lite+ classes (i.e. that WN synsets ?x ?y are relinkable to DOLCE-Lite+ classes), the relation "get-role-types" will find all the relations in DOLCE-Lite+ that are applicable to those classes, with a cardinality $\geq 1^{8}$. For example, given the previous example (2) of A-link, the classifier uses some of the DOLCE-Lite+ axioms to suggest the right conceptual relation. In fact, the WordNet synset art_exhibition\#1 is a (indirect) sub-class of the DOLCE class "unitary collection", a category for which the following axiom holds:

$$
\forall x \text {. Unitary_Collection }(x) \rightarrow \exists y \text {. Physical_Object }(y) \wedge \operatorname{Member}(x, y)
$$

Furthermore, since painting\#1 is a (indirect) sub-class of "physical object", and the axiom holds with a cardinality $\geq 1$, the classifier can propose the correct axiom.

In other cases, ABox-1 retrieves relations that are questionable. For example, given:

\section{A-link(boat\#1,travel\#1)}

we have no axiom like the following in DOLCE-Lite+:

$* \forall x$. Physical_Object(x) $\rightarrow \exists y$. Situation $(y) \wedge \operatorname{Setting}(x, y)$

then the A-link between boat and travel cannot be automatically classified and proposed as a "setting" one. In other words, in general it is not true that "for any physical object (boat\#1 is classified in the "object" branching) there is at least a situation (travel\#1 is classified within situations) as its possible "setting" (the relation holding between situations and other entities in DOLCE-Lite+): we can figure out objects in general, without setting them anywhere, at least within the scope of a computational ontology.

In other cases, there exist an appropriate relation, but it is applied in an incorrect way. For example, given:

\section{A-link(motor hotel\#1,parking area\#1)}

DOLCE-Lite finds the relation " $p$-spatial-location”, defined as (by using a KRSS-like syntax for description logics):

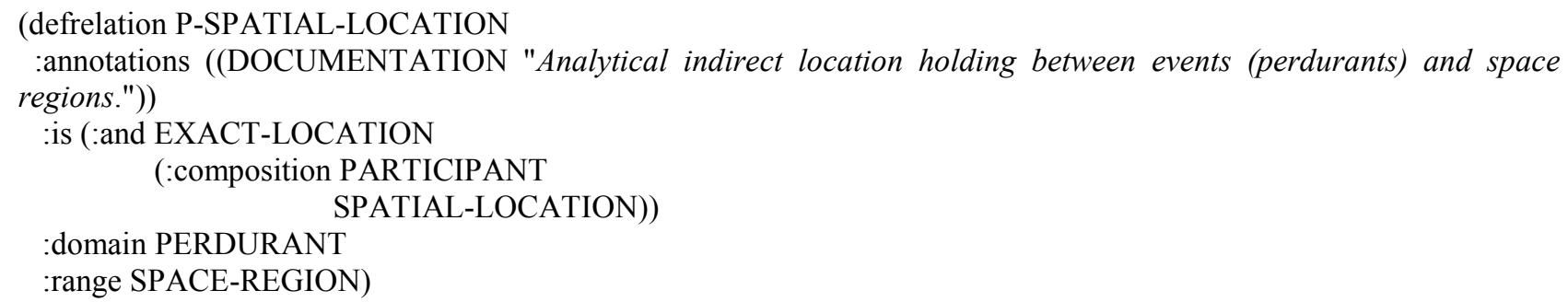

According to this intuitive and quite general definition, DOLCE-Lite+ suggests that motor hotel (a perdurant) is located in a parking area (space region), but here we have the opposite: the parking area is located in (belongs to) the motor hotel.

The above examples show that axioms representing generally acceptable intuitions in a foundational ontology may result inadequate in a given application domain, in which certain axiomatizations need an ad-hoc refinement.

The solution presented here exploits argument restrictions for the gloss axiomatization task. For this solution, we need a partition $\Pi$ of relation universes, according to the 25 valid pairs of argument restrictions that can be generated out of the five top categories of DOLCE-Lite+ (Object, Event, Quality, Region, and Situation), which on their turn constitute a partition on the domain of entities for DOLCE-Lite+. This enables us to assign one of the 25 relations to the A-link whose members are subsumed by the domain and range of that relation. For example, from:

$$
\begin{aligned}
& \operatorname{Boat}(x) \rightarrow \operatorname{Object}(x), \text { and } \\
& \text { Travel }(y) \rightarrow \operatorname{Situation}(y),
\end{aligned}
$$

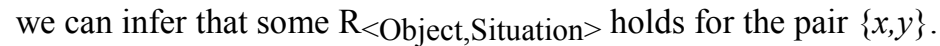

However, in DOLCE-Lite+, existing relations are based on primitives adapted from the literature, covering some basic intuitions and axiomatized accordingly. Therefore, the current DOLCE-Lite+ partition $\Pi \delta$ is not isomorphic with $\Pi$, while the same extensional coverage is supported. For example, the DOLCE-Lite+ relation "part" corresponds to a subset of the union of all the argument pairs in $\Pi$ that include only the same category (e.g., $<$ Event, Event $>$ ). $\Pi \delta$ is

\footnotetext{
${ }^{8}$ cardinality refers to the existential quantifier in $\mathrm{S}_{1}(x) \rightarrow \exists y . \mathrm{R}_{\text {ass }}(x, y) \wedge \mathrm{S}_{2}(y)$
} 
inadequate to perform an automatic learning of conceptual relations, because we cannot distinguish between "part" and other relations with the same universe (e.g. "connection"). Similarly, we cannot distinguish between different pairs of argument restrictions within the "part" universe (e.g. $<$ Event, Event $>$ vs. $<$ Object, Object $>$ ).

The choice of axioms in DOLCE-Lite + is motivated by the necessity of grounding the primitive relations in human intuition, for example in so-called cognitive schemata that are established during the first steps of an organism's life by interacting with its environment and using its specific abilities to react to the stimuli, constraints, and affordances provided by the context (Johnson 1987). In fact, without that grounding, the meaning of relations cannot be figured out at all (even though they are correct from a logical viewpoint).

There is also another reason for the inadequacy of $\Pi \delta$. A conceptual relation can be "mediated", e.g. manipulated through a composition (called also chaining, or joining in the database domain). For example, two events can be related not only because one is part of the other, but also because there can be someone participating in both events.

In brief: we cannot use $\Pi \delta$, since it does not discriminate at the necessary level of detail, and because it is not a partition at all, if we take into account mediated relations. On the other hand, we cannot use $\Pi$, because it is cognitively inadequate.

Consequently, we had to evolve a special partition $\Pi \delta+$ that keeps both worlds: a real partition, and cognitive adequacy. $\Pi \delta+$ denotes a partition with a precise mapping to $\Pi \delta$. In appendix 2 , the current state of $\Pi \delta+$ is shown.

For example, by using $\Pi \delta+$, the proposed relation for the car/engine example is (physical-)mereotopologicalassociation, defined as the union of some DOLCE-Lite+ primitive relations: part, connection, localization, constituency, etc., holding only within the physical object category. In fact, many possible relational paths can be walked from an instance of physical object to another.

Formally (in KRSS-like full syntax):

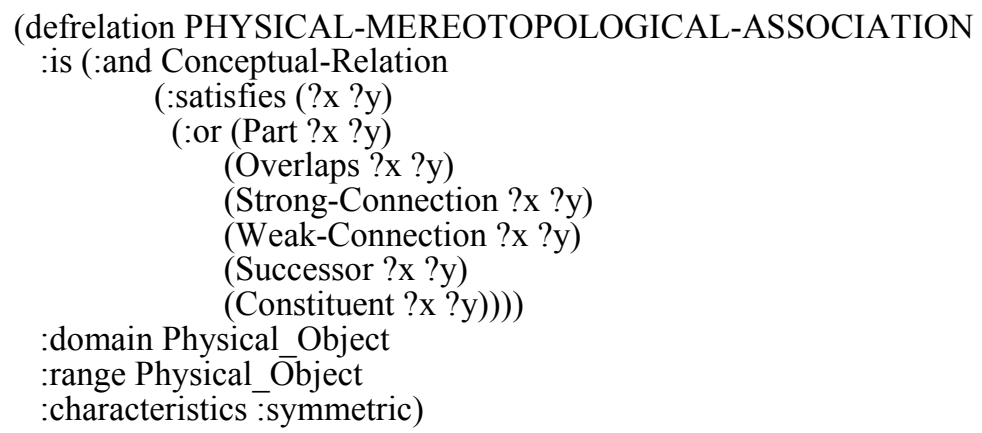

Starting from $\Pi \delta^{+}$, other relations have been defined for subsets of the domains and ranges of the relations in $\Pi \delta+$. By means of $\Pi \delta^{+}$, the query function can be adjusted as follows:

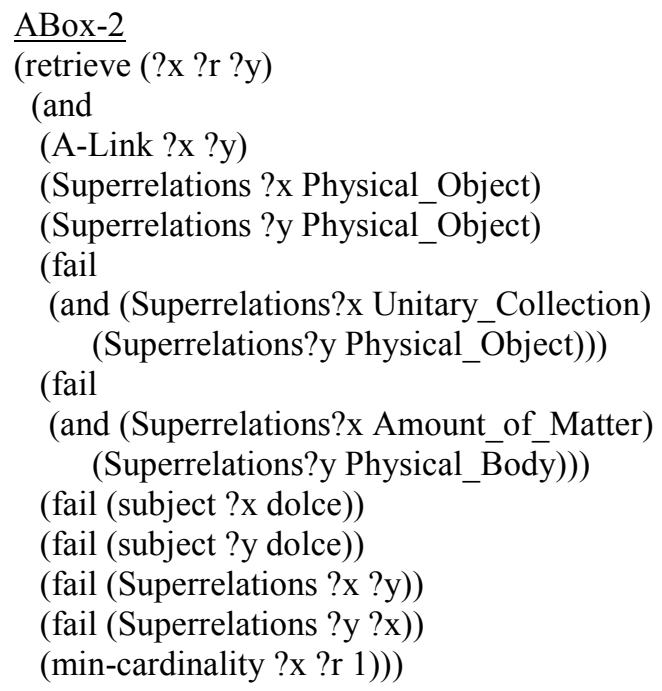

The query approximately reads "if two synsets subsumed by physical object (provided that the first is not an amount of matter or a collection, and that they are not related by hyperonymy), are linked by an A-link, tell me what relations in DOLCE+WordNet are applicable between those synsets with a cardinality of at least 1".

In this way, we are able to learn all the relations that are applicable to the classes ?x and ?y involved in the A-Link tuples. The intention here is, for example, to limit the universe of "Mereotopological_association", in order to give 
room to more specific relations, such as "member" or "constituent", with specialized universes. For example, applied to the synset car\#1 that has an A-link to the synset engine\#1, the query returns:

$$
\mathrm{R}_{\text {MEREOTOPOLOGICAL_ASSOCIATION }}(\text { car\#1,engine\#1) }
$$

which, on the basis of DOLCE-Lite+, is interpreted as an axiom on car\#l, stating that cars have a "physical mereotopological association" with an engine, because a DOLCE-Lite+ ancestor of both car\#1 and engine\#1 ("physical object") defines the universe of the relation mereotopological association with a cardinality of at least 1 on the range. This heuristics is equivalent to the logical axiom:

$$
\forall x . \operatorname{Car}(x) \rightarrow \exists y \text {. Engine }(y) \wedge \text { Mereotopological-Association }(x, y)
$$

Notice that at this level of generality, the classifier cannot infer the "component" relation that we intellectually guessed at the beginning of section 3.2. A more specific relation can be approximated, if we define more specialised relations and axioms. For example, a "functional co-participation" can be defined with a universe of only "functional objects", which are lower in the DOLCE-Lite+ hierarchy, but still higher than the pair of synsets associated by our automatic methodology of Section 3.1. Functional co-participation ("FCP") is defined by composing two participation relations with a common event (in the example, a common event could be "car running"):

$$
\operatorname{FCP}(x, y)=_{\mathrm{df}} \exists z . \text { Participant_in }(x, z) \wedge \operatorname{Participant}(y, z) \wedge \operatorname{Event}(z)
$$

FCP is closer to the "component" intuition. The last can be precisely inferred if we feed the classifier with core domain relations. For example, we may accept a domain rule that states that for all vehicles related to functional objects that play the role of system components for vehicles, the "component" relation holds:

$$
\begin{aligned}
\text { vehicles }^{\wedge} \operatorname{Component}(x, y)= & { }_{\mathrm{df}} \\
& \operatorname{FCP}(x, y) \wedge \operatorname{Vehicle}(x) \wedge \operatorname{Functional} \_\operatorname{Object}(y) \wedge \exists z . \operatorname{Plays}(y, z) \wedge
\end{aligned}
$$

In other words, by increasing the specificity of the domain (tourism in the examples discussed so far), we assume that relations should be specified accordingly. As discussed in this section, this process is triggered by the observation of some A-link, and proceeds semi-automatically until a reasonable coverage is reached.

Anyway, when the domain cannot be specified, even a generic association like "mereotopological" provides a better intuition than a bare A-link.

The conceptual relation partition is being incrementally verified, and the results of the experiment presented here can also be used as a test bed for creating a pruned set of domain-oriented relations. Notice that the pruned set of relations $\Pi \delta+$ is always consistent with the original DOLCE-Lite+ conceptual relations, with which the pruned relations form a larger intensional set (the extensional coverage is maintained).

\section{Experimental results and discussion}

The gloss disambiguation algorithm and the A-link interpretation methods have been evaluated on two sets of glosses: a first set of 100 general-purpose glosses ${ }^{9}$ and a second set of 305 glosses from a tourism domain. This allows us to evaluate the method both on a restricted domain and a non-specialized task.

For each term in a gloss, the appropriate WordNet sense has been manually assigned by two annotators, for over 1000 words.

To assess the performance of the gloss disambiguation algorithm we used two common evaluation measures: recall and precision. Recall provides the percentage of right senses with respect to the overall number of terms contained in the examined glosses. In fact, when the disambiguation algorithm terminates, the list $P$ may still include terms for which no relation with the synsets in $D$ could be found. Precision measures the percentage of right senses with respect to the retrieved gloss senses. A baseline precision is also computed, using the "first sense choice" heuristic. In WordNet, synsets are ordered by probability of use, i.e. the first synset is the most likely sense. For a fair comparison, the baseline is computed only on the words for which the algorithm could retrieve a synset.

Table 1 gives an overview of the results. Table 1a provides an overall evaluation of the algorithm, while table $1 \mathrm{~b}$ computes precision and recall grouped by morphological category. The precision is quite high (well over $90 \%$ for both general and domain glosses) but the recall is around 40\%. Remarkably, the achieved improvement in precision with respect to the baseline is much higher for general glosses than for domain glosses. This is motivated by the fact that

\footnotetext{
${ }^{9}$ The 100 general glosses have been randomly selected among the 705 glosses used to re-link WordNet to the DOLCE-lite upper ontology.
} 
general glosses include words that are more ambiguous than those in domain glosses. Therefore the general gloss baseline is quite low. This means also that the disambiguation task is far more complex in the case of general glosses, where our algorithm shows particularly good performance.

An analysis of performance by morphological category (Table 1b) shows that noun disambiguation has much higher recall and precision. This is motivated by the fact that, in WordNet, noun definitions are richer than for verbs and adjectives. The WordNet hierarchy for verbs is known as being more problematic with respect to nouns. In the future, we plan to integrate in our algorithm verb information from FRAMENET $^{10}$, a lexico-semantic knowledge base providing rich information especially for verbs.

\begin{tabular}{|l|l|l|l|l|l|l|l|}
\hline Domains & \multicolumn{1}{|c|}{ \# glosses } & \multicolumn{1}{|c|}{ \# words } & $\begin{array}{c}\text { \# disamb. } \\
\text { words }\end{array}$ & \# of which ok & Recall & \multicolumn{1}{c|}{ Precision } & $\begin{array}{c}\text { Baseline } \\
\text { Precision }\end{array}$ \\
\hline Tourism & 305 & 1345 & 636 & 591 & $47,28 \%$ & $92,92 \%$ & $82,55 \%$ \\
\hline Generic & 100 & 421 & 173 & 166 & $41,09 \%$ & $95,95 \%$ & $67,05 \%$ \\
\hline
\end{tabular}

\begin{tabular}{|l|l|l|l|l|l|l|l|l|l|}
\hline Domains & \multicolumn{1}{|c|}{$\begin{array}{c}\text { noun } \\
\text { recall }\end{array}$} & $\begin{array}{c}\text { noun } \\
\text { precision }\end{array}$ & adj recall & $\begin{array}{c}\text { adj } \\
\text { precision }\end{array}$ & verb recall & $\begin{array}{c}\text { verb } \\
\text { precision }\end{array}$ & $\begin{array}{c}\text { \# tot } \\
\text { nouns in } \\
\text { glosses }\end{array}$ & $\begin{array}{c}\text { \# tot adj in in tot verbs } \\
\text { glosses }\end{array}$ & $\begin{array}{c}\text { \# glosses } \\
\text { in gloss }\end{array}$ \\
\hline Tourism & $64,52 \%$ & $92,86 \%$ & $28,72 \%$ & $89,29 \%$ & $9,18 \%$ & $77,78 \%$ & 868 & 195 & 294 \\
\hline Generic & $58,27 \%$ & $95,95 \%$ & $28,38 \%$ & $95,24 \%$ & $5,32 \%$ & $80 \%$ & 254 & 74 & 94 \\
\hline
\end{tabular}

Table 1a) performance of the gloss disambiguation algorithm b) performance by morphological category.

In Table 2 we summarize the efficacy of the A-link semi-automatic axiomatization, after the partly manual creation of a domain view $\Pi \delta+$ as discussed in section 3.2.

\begin{tabular}{|l|l|l|l|l|l|l|l|}
\hline Domains & Synsets & A-links & Noun-only & Subsumptions & Filtered A-links & Axioms generated & $\begin{array}{l}\text { Correct } \\
\text { (best arrangement) }\end{array}$ \\
\hline Tourism & 305 & 725 & 644 & 209 & 435 & 569 & 511 \\
\hline Generic & 100 & 212 & 187 & 40 & 147 & 142 & 121 \\
\hline
\end{tabular}

Table 2. Axiomatizations for the A-links.

As a preventive measure, we have excluded the A-links that include either an adjective or a verb, since these synsets have not been yet integrated with DOLCE-Lite+. Another measure excluded the A-links that imply a subsumption (subclass) link, since these are already formalized. This filter has been implemented as a simple ABox query that uses relations that range on classes:

$\underline{\mathrm{ABox}-3}$

(retrieve (?x ?y) (and (A-Link ?x ?y) (Superrelations ?x ?y)))

These measures reduced the amount of A-Links from the experimental set to $582(435+147)$. We have used these tuples to run the revised query ABox-2.

The revised query produced $711(569+142)$ candidate axioms by using all the pruned relations defined for the experiment. Table 3 shows the resulting axioms ordered by generality of the relation universes (domain and range).

\begin{tabular}{|l|l|l|l|l|}
\hline & Tourism & Tourism correct & Generic & Generic correct \\
\hline Total amount of axioms & 569 & $511(89.80 \%)$ & 142 & $121(85.21 \%)$ \\
\hline Axioms with generic universes & 540 & $490(90.74 \%)$ & 139 & $121(87.05 \%)$ \\
\hline Axioms with some specific universes & 545 & $507(93.02 \%)$ & 136 & $118(86.76 \%)$ \\
\hline Axioms with only topmost universes & 375 & $356(94.93 \%)$ & 110 & $98(89.09 \%)$ \\
\hline
\end{tabular}

Table 3. Axiomatizations ordered by generality.

\footnotetext{
${ }^{10} \mathrm{http}: / /$ www.icsi.berkeley.edu/ framenet/
} 
The most relevant results are:

- One third of the A-Links from the tourism domain are actually subsumption links, while only $20 \%$ from the mixed generic set is a subsumption. This could be explained by the fact that glosses for generic synsets are less informative, or because generic words are not defined, in $\mathrm{WN}$, in terms of more generic ones.

- The correct subset of axioms learnt for the tourism domain is about 4 to $6 \%$ larger than for the generic one with reference to the whole sets.

- We have tried to use some relations that are in principle "less precise". For example, a universe composed of physical objects and amounts of matter has a basic intuition of "constituency", and the relation has_n_constituent has been defined to such purpose. This relation has proved very inefficient though: in the generic set, only $50 \%$ of learnt axioms are correct, while in the tourism domain, only $16 \%$ are correct. We could expect that domains like earth science and physics can be more appropriate for constituency relations. For this reason, we have included a relation with a functional flavor in the experimental set of relations (including $\Pi \delta+$ and its specializations), called "provides", and defined on functional objects and functional matters (this universe is a meaningful subset of the previous one). This relation proved quite efficient in the tourism domain, just as expected, with about $78 \%$ of correct axioms, while it is useless in the generic set, with $0 \%$. An example of "provides" axioms is the following: $\forall x$. $\operatorname{Brasserie}(x) \rightarrow \operatorname{Beer}(y) \wedge \operatorname{Provides}(x, y)$.

This, and similar examples, confirm our expectations about the importance of developing dedicated sets of relations for different domains or tasks, while a "ground" level of relations is useful everywhere: in fact, the percentage of correct axioms increases if only the first level of the relation hierarchy is taken into account ( $95 \%$ in tourism, $89 \%$ in generic).

- In 8 cases, the axioms were not definable with a cardinality $\geq 1$, although they could be used in more restricted domains or for subclasses of the universe.

- Some indirect A-links can be investigated as well (though our first strategy has been to disregard indirect links, as explained in section 3.1). For example in the retrospective\#1 example of Figure 2, two synsets (painting\#1 and statue\#1) are learnt as "indirect" synsets (they are learnt from the glosses relative to the hyperonyms of retrospective\#1). But paintings and statues are not always found in exhibitions, then we are not allowed to infer an axiom with cardinality $\geq 1$. In these cases, the algorithm could be refined to propose an axiom that includes a common parent to both painting\#1 and statue\#1, i.e. art\#1, which incidentally is another "indirect" A-link to retrospective\#1. In Figure 5 the refined A-links for retrospective\#1 are shown: a retrospective in WordNet 1.6 has the intended meaning of a (unitary) collection in DOLCE-Lite+, which is a kind of non-agentive functional object. This lets the classifier infer:

- a "functional association" to artist\#1, because an artist is a functional role;

- a more precise "plays" relation to life\#12, since an artistic biography is a functional role as well, and a collection of art works plays just the role of an artistic biography;

- a subsumption of retrospective\#1 by exhibition\#2;

- three "has_member" relations to the indirect A-links: art\#1, painting\#1, and statue\#1. These are correct, since a collection can have functional objects (art works) as members. But while the first has a meaningful cardinality 1 to $\mathrm{n}$, the others have a logically irrelevant cardinality of 0 to $\mathrm{n}$.

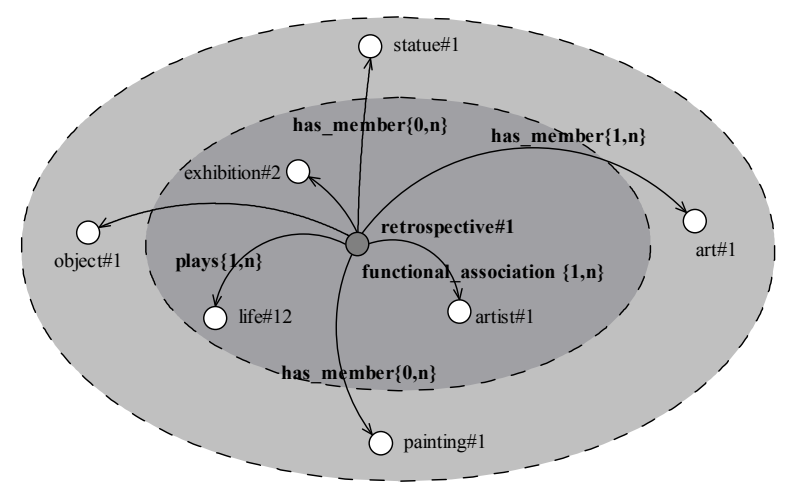

Figure 5. Interpretation of A-links for retrospective\#1.

\section{Conclusions}

In this paper we presented preliminary results of OntoWordNet, a large scale project aiming at the "ontologization" of WordNet 1.6. We presented a two step methodology: during the first, automatic phase, natural language word sense 
glosses in WordNet are parsed, generating a first, approximate definition of WN concepts (called synsets). In this definition, generic associations (A-links) are established between a concept and the concepts that co-occur in its gloss.

In a second phase, the foundational top ontology DOLCE (in the DOLCE-Lite+ version), including few hundreds formally defined concepts and conceptual relations, is used to interpret A-links in terms of axiomatised conceptual relations. This is a partly automatic technique that involves generating solutions on the basis of the available axioms, and then creating a specialized partition of the axioms (the set $\Pi \delta+$ and its specializations) in order to capture more domain-specific phenomena.

Overall, the experiments that we conducted show that a high performance may be obtained through the use of automatic techniques, significantly reducing the manual effort that would be necessary to pursue the objective of the OntoWordNet project.

\section{References}

(Basili et al. 1996) Basili R., Pazienza M.T. and Velardi P. An Empirical Symbolic Approach to Natural Language Processing, Artificial Intelligence, n. 85, pp.59-99, (1996).

(Berners-Lee, 1998) Berners-Lee T., Semantic Web Road map, http://www.w3.org/DesignIssues/Semantic.html, 1998 (Fellbaum 1995) Fellbaum, C. WordNet: an electronic lexical database, Cambridge, MIT press, (1995).

(Gangemi et al. 2001) Gangemi, A., Guarino, N., Masolo, C., and Oltramari, A. 2001. Understanding top-level ontological distinctions. In Proceedings of IJCAI-01 Workshop on Ontologies and Information Sharing. Seattle, USA, AAAI Press: 26-33. http://SunSITE.Informatik.RWTHAachen. DE/Publications/CEUR-WS/Vol-47/

(Gangemi et al. 2002) Gangemi A., Guarino N. Masolo C. Oltramari A., Schneider L. "Sweetening Ontologies with DOLCE", Proc. Of EKAW02 http://citeseer.nj.nec.com/cache/papers/cs/26864/http

(Harabagiu and Moldovan 1999) Harabagiu S. and Moldovan D. Enriching the WordNet Taxonomy with Contextual Knowledge Acquired from Text. AAAI/MIT Press, (1999).

(Karkaletsis V. Cucchiarelli A Paliouras G. Spyropolous C. Velardi P. "Automatic adaptation of Proper Noun Dictionaries through cooperation of machine learning and probabilistic methods" 23rd annual ACM-SIGIR, Athens, June 2000

(Johnson 1987) Johnson, Mark. 1987. The Body in the Mind. Chicago: University of Chicago Press

A. Maedche and S. Staab. Ontology learning for the Semantic Web. IEEE Intelligent Systems, 16(2), 2001.

(Mac Gregor, 1993) MacGregor, R. M. 1991. Using a Description Classifier to

Enhance Deductive Inference. In Proceedings of Seventh IEEE Conference on AI Applications: 141-147.

(Masolo et al. 2002) Claudio Masolo, Stefano Borgo, Aldo Gangemi, Nicola Guarino, Alessandro Oltramari, and Luc Schneider. The WonderWeb Library of Foundational Ontologies. WonderWeb Deliverable 17, 2002.

(Magnini and Caviglia 2000) Magnini, B. and Cavaglia, G.: Integrating Subject Field Codes into WordNet. Proceedings of the 2nd International Conference on Language resources and Evaluation, LREC2000, Atenas .

(Miller et al. 1993) G. A. Miller, R. Beckwith, C. Fellbaum, D. Gross \& K. Miller; "Introduction to WordNet: An On-Line Lexical Database"; http://www.cosgi.princeton.edu/ wn; August 1993.

(Mihalcea and Moldovan, 2001) Milhalcea, R. and Moldovan. D. eXtended WordNet: progress report. NAACL 2001 Workshop on WordNet and Other Lexical Resources, Pittsbourgh, June (2001).

(Moeller, 2001) Volker Haarslev, Ralf Möller Description of the RACER System and its Applications Proceedings International Workshop on Description Logics (DL-2001), Stanford, USA, 1.-3. August 2001

(Morin, 1999) Morin E., Automatic Acquisition of semantic relations between terms from technical corpora, Proc. of $5^{\text {th }}$ International Congress on Terminology and Knowledge extraction, TKE-99, (1999).

(Navigli et al. 2003) Navigli,R P. Velardi and A. Gangemi, Ontology Learning and its Application to Automated Terminology Translation, IEEE Intelligent Systems, vol. 18, n.1, pp. 22-31, January 2003

(Searle, 1985) Searle, J.R and Vanderveken, D. Foundations of Illocutionary Logics, Cambridge University Press, (1985).

(Smith and Welty, 2001) Smith, B. and Welty, C. Ontology: towards a new synthesis, Formal Ontology in Information Systems, ACM Press, (2001). 
(Vossen 2001) Vossen P. Extending, Trimming and Fusing WordNet for technical Documents, NAACL 2001 workshop on WordNet and Other Lexical Resources, Pittsbourgh, July (2001).

\section{Web Sites}

DAML+OIL http://www.daml.org/2001/03/daml+oil-index

LDC corpus http://www.ldc.upenn.edu/

FRAMENET http://www.icsi.berkeley.edu/ framenet/

WordNet 1.6 http://www.cogsci.princeton.edu/ wn/w3wn.html

Semcor http://engr.smu.edu/ rada/semcor/

WonderWeb http://wonderweb.semanticweb.org

\section{Appendix 1}

Dolce top ontology of concepts and relations

\begin{tabular}{|c|c|}
\hline ENTITY & Anything conceivable \\
\hline : QUALITY-SPACE & Any space of values (e.g. dimensional spaces) \\
\hline :: REGION & Any value or sedt of values \\
\hline$:::$ ABSTRACT-REGION & Any set of non-physical or temporal values (e.g. monetary) \\
\hline$:::$ PHYSICAL-REGION & Any set of physical values (e.g. volume, color) \\
\hline$::::$ SPACE-REGION & Any set of spatial values (e.g. geographic coordinates) \\
\hline : : : TEMPORAL-REGION & Any set of temporal values (e.g. gregorian date system) \\
\hline : QUALITY & Any individual counterpart of a region (e.g. the color of a rose) \\
\hline : ENDURANT ( $\approx$ Object) & Any entity with a direct spatial value (localization), cf. Object \\
\hline$::$ NON-PHYSICAL-ENDURANT & Any non-physical object, such as social or mental objects \\
\hline$:::$ S-DESCRIPTION & Any reified conceptualization or theory (e.g. plans, norms) \\
\hline : : : COURSE & Any (abstract) sequence of activities (cf. process model) \\
\hline$:::$ FUNCTIONAL-ROLE & Any role played by an object (e.g. minister, student) \\
\hline ::: PARAMETER & Any selection of value sets (e.g. speed limits) \\
\hline $\begin{array}{l}: \quad: \quad \text { PHYSICAL-ENDURANT } \\
(\approx \text { Substance })\end{array}$ & Any physical entity with a direct localization, cf. Substance \\
\hline$:::$ AMOUNT-OF-MATTER & Any amount of matter without a unity (e.g. sand, milk) \\
\hline$::::$ FUNCTIONAL-MATTER & Any amount of matter according to scope (e.g. food) \\
\hline$:::$ FEATURE & Any relevant part or place within an object (e.g. edges, holes) \\
\hline$:::$ PHYSICAL-OBJECT & Any substance with a unity criterion (e.g. stones, livers, roses) \\
\hline$::::$ AGENTIVE-PHYSICAL-OBJECT & Any physical object with intentionality (e.g. organisms, robots) \\
\hline $\begin{array}{l}:::::: \text { AGENTIVE-FUNCTIONAL- } \\
\text { OBJECT }\end{array}$ & Any agentive object according to some scope (e.g. robots) \\
\hline $\begin{array}{l}:::: \text { NON-AGENTIVE-PHYSICAL- } \\
\text { OBJECT }\end{array}$ & Any physical object without intentionality (e.g. stones, livers) \\
\hline 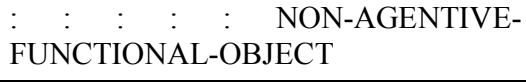 & $\begin{array}{l}\text { Any physical object according to some scope but with no intentionality (e.g. } \\
\text { hammers, walls) }\end{array}$ \\
\hline : PERDURANT ( $\approx$ Event) & Any entity with a direct temporal value (temporal presence) \\
\hline$::$ EVENT & Any temporal entity with heterogeneous parts (e.g. activities, phenomena) \\
\hline : : STATE & Any temporal entity with homogeneous parts \\
\hline : SITUATION & $\begin{array}{l}\text { Any localized counterpart of a S-description (e.g. conditions, environments, } \\
\text { states of affaris, observed facts) }\end{array}$ \\
\hline
\end{tabular}

\begin{tabular}{|l|l|}
\hline CONCEPTUAL-RELATION & Entity $(x)$, Entity(y). The top-level relation between entities whatsoever. \\
\hline : IMMEDIATE-RELATION & Any relation holding directly, without any other intermediate relation chaining \\
\hline$::$ CONSTITUENT & $\begin{array}{l}\text { A relation of constituency between e.g. matter and objects, e.g. skin made up of } \\
\text { epithelial tissue }\end{array}$ \\
\hline$::$ HAS-MEMBER & $\begin{array}{l}\text { A constituency between collections and their members, e.g. a society and its } \\
\text { members }\end{array}$ \\
\hline$:::$ SETTING-FOR & A constituency between situations and their entities, e.g. $a$ flu and its observed \\
\hline
\end{tabular}




\begin{tabular}{|c|c|}
\hline & symptoms \\
\hline$::$ HOST & $\begin{array}{l}\text { Feature (x), Physical-Endurant(y). The relation between features and objects, e.g. } \\
\text { a hole in the cheese }\end{array}$ \\
\hline$::$ INHERENT-IN & $\begin{array}{l}\text { Quality }(x), \text { Entity }(y) \text {. The relation between qualities and entities, e.g. the red of a } \\
\text { rose }\end{array}$ \\
\hline$::$ PART & Any part relation (but not constituency), e.g. a chair and its legs \\
\hline ::: PROPER-PART & Any antisymmetric part, e.g. a human body and its legs \\
\hline$::::$ BOUNDARY & A part relation between an entity and its boundary, e.g. Italy's borders \\
\hline$::::$ COMPONENT & A functional part relation, e.g. a car and its parts \\
\hline :: PARTICIPANT & Event $(x)$, Object $(y)$, the relation for taking part in something, e.g. love and lovers \\
\hline$::$ Q-LOCATION & $\begin{array}{l}\text { Quality(x), Quality-Space(y), the relation between qualities and their } \\
\text { counterparts, e.g. the red of a rose and its representation in a color palette }\end{array}$ \\
\hline$::$ REFERENCES & $\begin{array}{l}\text { S-description(x), Situation(y), the relation between conceptualizations and } \\
\text { situations, e.g. a plan and an activity executed according to that plan }\end{array}$ \\
\hline$:::$ PLAYED-BY & $\begin{array}{l}\text { Functional-role(x), Object(y), the relation for role-playing, e.g. student and a } \\
\text { person who is enlisted in a university }\end{array}$ \\
\hline$::$ WEAK-CONNECTION & A generic, unordered connection \\
\hline$::$ PREDECESSOR & An ordered connection, e.g. the relation between two consecutive intervals \\
\hline : MEDIATED-RELATION & $\begin{array}{l}\text { Any relation holding indirectly, for which some other relation must hold } \\
\text { preliminarily }\end{array}$ \\
\hline$::$ CO-PARTIPATION & $\begin{array}{l}\text { Object }(x), \text { Object }(y), \text { the relation holding between two objects that participate in } \\
\text { th same event or state }\end{array}$ \\
\hline$\because:$ GENERIC-LOCATION & Any relation between entities whatsoever \\
\hline$:::$ EXACT-LOCATION & $\begin{array}{l}\text { Any location between objects or events, and a region, e.g. Rome and its } \\
\text { geographic coordinates }\end{array}$ \\
\hline$::::$ PHYSICAL-LOCATION & $\begin{array}{l}\text { Any location between physical objects, and physical regions, e.g. a stone and its } \\
\text { volume }\end{array}$ \\
\hline$::::$ SPATIAL-LOCATION & $\begin{array}{l}\text { Any location between physical objects, and spatial regions, e.g. a planet and its } \\
\text { astral coordinates }\end{array}$ \\
\hline$::::$ TEMPORAL-LOCATION & $\begin{array}{l}\text { Any location between events, and temporal regions, e.g. a football match and its } \\
\text { scheduling, or its duration }\end{array}$ \\
\hline$:::$ APPROXIMATE-LOCATION & Any location between entities other than regions, e.g. the pen is on the table \\
\hline
\end{tabular}

\section{Appendix 2}

The experimentally derived set of relations (including $\Pi \delta^{+}$and its specializations)

\begin{tabular}{|c|c|c|c|c|}
\hline & \begin{tabular}{|l} 
Touris \\
m
\end{tabular} & \begin{tabular}{|l|}
$\begin{array}{l}\text { Tourism } \\
\text { correct }\end{array}$ \\
\end{tabular} & $\begin{array}{l}\text { Generi } \\
\text { c }\end{array}$ & $\begin{array}{l}\text { Generic } \\
\text { correct }\end{array}$ \\
\hline \multicolumn{5}{|l|}{ Conceptual_Relation (Entity, Entity) } \\
\hline :Descriptive_Association (Object, S-Description) & 7 & 6 & 5 & 4 \\
\hline : Descriptive_Constituent_Of(Functional-Role, S-Description) & & & 1 & 0 \\
\hline :Functional_Association (Object, Functional-Role) & 72 & 68 & 22 & 19 \\
\hline : Functional_Role_Co_Participation (F-Role,F-Role) & 21 & 21 & 13 & 12 \\
\hline :Has_Physical_Location (Physical-Object, Geographical-Entity) & 6 & 3 & & \\
\hline : Has_Functional_Location (Functional-Object, Geographical-Entity) & 6 & 3 & & \\
\hline :Has_Quality_Region (Object, Region) & 9 & 8 & 2 & 0 \\
\hline : Host_Of (Physical-Object, Feature) & 7 & 2 & 3 & 2 \\
\hline \multicolumn{5}{|l|}{ :Inherent_In (Quality, Entity) } \\
\hline :Mereotopological_Association (Physical-Object, Physical-Object) & 140 & 140 & 29 & 29 \\
\hline $\begin{array}{l}:: \text { Agentive_CoParticipation (Agentive-Physical-Object, Agentive-Physical- } \\
\text { Object) }\end{array}$ & 1 & 1 & 2 & 1 \\
\hline \multicolumn{5}{|l|}{\begin{tabular}{|l|l}
$:$ : Biological_Part (Organism, Biological_Object) & \\
\end{tabular}} \\
\hline : : Functional_CParticipation (Functional-Object, Functional-Object) & 98 & 94 & 1 & 1 \\
\hline$:$ : Has Member (Collection, Object) & 4 & 4 & & \\
\hline
\end{tabular}




\begin{tabular}{|c|c|c|c|c|}
\hline \multirow{2}{*}{\multicolumn{5}{|c|}{$\begin{array}{l}\text { : Material_CoParticipation Functional-Matter, Functional-Matter) } \\
\text { : Material_Constituent_Of (Amount-Of-Matter, Physical-Object) }\end{array}$}} \\
\hline & & & & \\
\hline : Provides (Functional-Object, Functional-Matter) & 22 & 17 & 3 & 0 \\
\hline \multicolumn{5}{|l|}{ : Uses_CoParticipation (Agentive-Physical-Object, Functional-Object) } \\
\hline Participant (Event, Object) & 14 & 14 & & \\
\hline : Agentive Participant (Event, Agentive-Object) & 3 & 3 & & \\
\hline P_Has_Quality_Region (Event, Region) & 1 & 1 & & \\
\hline Setting_For (Situation, Entity) & 18 & 17 & & \\
\hline :Referenced_By (Situation, S-Description) & 1 & 0 & & \\
\hline Temporal_Mereotopological_Association (Event, Event) & 6 & 5 & 2 & 1 \\
\hline \multicolumn{5}{|l|}{ nv_Conceptual_Relation (Entity, Entity) } \\
\hline Host (Feature, Physical-Object) & 1 & 1 & & \\
\hline :Inherence_Of (Entity, Quality) & 2 & 0 & 4 & 4 \\
\hline Inv_Descriptive_Association (S-Description, Object) & 7 & 7 & 4 & 4 \\
\hline : Has_Descriptive_Constituent (S-description, Functional-Role) & 1 & 0 & & \\
\hline :Inv_Functional_Association (Object, Functional-Role) & 45 & 45 & 21 & 19 \\
\hline \multicolumn{5}{|l|}{ Inv_Mereotopological_Association (Physical-Object, Physical-Object) } \\
\hline : : Biological_Part_Of (Biological-Object, Organism) & & & 4 & 4 \\
\hline : Has_Material_Constituent (Physical-Object, Amount-Of-Matter) & 24 & 4 & 6 & 3 \\
\hline :Used_By_Co_Pcp (Functional-Object, Agentive-Physical-Object) & 7 & 4 & & \\
\hline : Member_Of (Object, Collection) & 1 & 0 & & \\
\hline \multicolumn{5}{|l|}{ : Provided_By (Functional-Matter, Functional-Object) } \\
\hline \multicolumn{5}{|l|}{ Inv_Temporal_Mereotopological_Association (Event, Event) } \\
\hline : Participant_In (Object, Event) & 14 & 13 & 6 & 6 \\
\hline : Agentive_Participant_In (Functional-Object, Event) & 1 & 1 & & \\
\hline :Physical_Location_Of (Geographical-Entity, Physical-Object) & 2 & 2 & 2 & 2 \\
\hline :Functional_Location_Of (Geographical-Entity, Functional-Object) & 1 & 1 & & \\
\hline :Quality_Region_Of(Region, Object) & 3 & 3 & 2 & 1 \\
\hline \multicolumn{5}{|l|}{ Quality_Region_Of_P (region, Event) } \\
\hline : Setting (Entity, Situation) & 21 & 21 & 8 & 7 \\
\hline$:$ : References (S-Description, Situation) & 3 & 2 & 2 & 2 \\
\hline
\end{tabular}

